\title{
Molecular profile reveals immune-associated markers of medulloblastoma for different subtypes
}

\section{Shenglan Li}

beijng tiantan hospital

\section{Zhuang Kang}

beijng tiantan hospital

\section{Jinyi Chen}

beijng tiantan hospital

\section{Can Wang}

beijng tiantan hospital

\section{Zehao Cai}

beijng tiantan hospital

\section{Leixin Pan}

Beijing Institute of Technology

\section{Feng Chen}

beijng tiantan hospital

\section{lishenglan}

beijng tiantan hospital

liwenbin ( $\nabla$ liwenbin@ccmu.edu.cn)

beijng tiantan hospital

\section{Research Article}

Keywords: MethyICIBERSORT, immune infiltration, WGCNA, PPI, Medulloblastoma

Posted Date: August 16th, 2021

DOI: https://doi.org/10.21203/rs.3.rs-816357/v1

License: (c) (1) This work is licensed under a Creative Commons Attribution 4.0 International License.

Read Full License 


\section{Abstract}

Medulloblastoma is a common intracranial tumor among children. In recent years, research on cancer genome has established four distinct subtypes of medulloblastoma: WNT, SHH, Group3, and Group4. Each subtype has its own transcriptional profile, methylation changes, and different clinical outcomes. Treatment and prognosis also vary depending on the subtype. Based on the methylation data of medulloblastoma samples, methyICIBERSORT was used to evaluate the level of immune cell infiltration in medulloblastoma samples and identified 10 kinds of immune cells with different subtypes. Combined with the immune database, $293 \mathrm{Imm}$-DEGs were screened. Imm-DEGs were used to construct the coexpression network, and the key modules related to the level of differential immune cell infiltration were identified. Three immune hub genes (GAB1, ABL1, CXCR4) were identified according to the gene connectivity and the correlation with phenotype in the key modules, as well as the PPI network involved in the genes in the modules. The subtype marker was recognized according to the immune hub, and the subtype marker was verified in the external data set, Finally, the methylation level of immune hub gene among different subtypes was compared and analyzed, at the same time, tissue microarray was used for immunohistochemical verification, and a multi-factor regulatory network of hub gene was constructed. Identifying subtype marker is helpful to accurately identify the subtypes of medulloblastoma patients, and can accurately evaluate the treatment and prognosis, so as to improve the overall survival of patients.

\section{Introduction}

Medulloblastoma (MB) is considered to be a highly malignant and fast-growing brain tumor among pediatric central nervous system malignancies, accounting for $8-10 \%$ of all pediatric brain tumors and is becoming the most common type [1]. Classification of medulloblastoma was primarily based on histopathology, while with recent developments in high throughput transcriptome data analyses, subclassification of medulloblastomas applied by a transcriptional approach can be achieved[2]. In 2010, four major subgroups of medulloblastoma distinguished by advanced genomic research were named as follow: wingless-activated (WNT-MB), sonic-hedgehog-activated (SHH-MB), Group 3, and Group 4, each characterized by unique genetic alterations, transcriptional/methylation profiles, and clinical outcomes [3]. These four working groups are now considered to be individual biological entities.

WNT-MBs are the most well-known subgroup of MB with an activation of the WNT pathway and harbors mutations in the exon 3 of CTNNB1 and monosomy chromosome 6 . Indeed, the long-term survival rates for patients with WNT-MBs are likely to exceed $90 \%$, mostly patients often die as a consequence of complications of therapy or secondary neoplasms rather than from recurrent WNT medulloblastomas [4]. SHH-WBs, with an activation of the SHH pathway, have largely been distinguished based on transcriptional profiling. Immunohistochemical staining for SFRPI, or GAB1, and deletion of chromosome $9 q$ are other approaches to distinguish SHH-MBs. The overall prognosis of SHH-MBs is similar to that of Group 4-MBs, between the WNT-MBs (good) and Group 3-MBs (poor) [3]. Group 3 has a high metastatic rate which implies poorer prognoses, particularly in those with amplified MYC $[5,6]$. Group 4 is the most 
prevalent type of medulloblastoma but its molecular pathogenesis is not well understood [7]. Patients with absent chromosome 11 have an excellent clinical outcome (exceeding 90\%). While, comparing with WNT- or SHH-MBs, adults with Group 4-MBs have a significantly worse prognosis[8]. These subgroups with specific genetic alterations and clinical outcomes indicated that tumors with similar transcriptomes may behave in a similar biological manner, providing direction for molecular targeted therapy and clinical risk stratification.

The tumor immune microenvironment (TIME) is of major importance to the evolution of tumor and can modulate the response to chemo- and radiotherapy[9]. However, current immune therapies, including cancer vaccinations, chimeric antigen receptor T-cell (CAR-T) therapy, and immune checkpoint inhibitors (ICIs) do not really benefit all the patients[10]. Therefore, exploring the interaction between TIME and tumor provides the basis for taking full advantage of the potential of immunotherapy.

Previous results that were based on preclinical animal models or an immunohistology profiling of tumors are limited in sample size and struggle to meet statistical requirements. Furthermore, the existing markers for MB subgroups classification are not highly indicative of immune infiltration. With the development of high-throughput data analysis, large gene expression datasets of medulloblastoma have been constructed, including one dataset of 763 tumors samples [11], which could be used to dissect the molecular composition of TIME and elucidate the pathogenesis by computational methods.

Hence, the TIME of medulloblastoma was investigated using gene expression profiles and the differential expressed gene related to immune gene was recognized. The deconvolution algorithm was adopted to analyze tumor-infiltrating the immune cells among MB subgroups and WGCNA was used to construct a co-expression network. The hub genes of the immune infiltration of medulloblastoma were identified first, a multi-factor regulatory network was constructed based on the hub genes to provide a new perspective for tumor immune microenvironment toward medulloblastomas. In this study, different subtypes of immune infiltrating cells and immune related genes were screened for the first time to fully reflect the immune cell infiltration of MB. Our results are expected to promote the immunotherapy and individualized treatment of MB.

\section{Method And Materials}

\subsection{Patients and data collection}

All medulloblastoma gene expression profiles were acquired from the Gene Expression Omnibus (GEO) database and NCBI's publicly available genomics database. Dataset GSE85218 contains DNA methylation and gene expression profiling of primary medulloblastoma samples from the same public cohort. Gene expression profiling of medulloblastomas was from dataset GSE85217 across 763 primary samples, based on the platform GPL22286. DNA methylation profiling of 763 primary samples were from dataset GSE85212, based on platform GPL13534. Both GSE85217 and GSE85212 are sub-series belonging to the super series GSE85218. Those 763 intertumoral heterogenic primary samples comprise four distinct entities: WNT $(n=70), S H H(n=223)$, Group $3(n=144)$, and Group $4(n=326)$. The immune 
gene profiles were downloaded from the ImmPort database (http://www.immport.org/, 36 Notes released, September 2020) and the InnateDB (http://www.innatedb.ca/, Version 5.4), which contained 2533 immune genes.

2.2 Analysis of tumor-infiltrating immune cell among MB subgroups.

CIBERSORT is a support vector regression modeling to gene expression microarray data, developed to permit the in-silico deconvolution of complex cellular mixtures and to estimate tumor purity.

MethyICIBERSORT is a CIBERSORT-based deconvolution to DNA methylation profile of tumor tissue and is also able to estimate the degree of immune cell infiltration. Analysis of variance was used to compare the degree of immune cells infiltration among different subgroups and to identify the differential immune infiltrating cells. $\mathrm{P}<0.05$ was considered to be statistically significant.

2.3 The immune differential expressed genes (imm-DEGs) among MB subgroups.

Combined with the immune gene profile, limma package in the R software was applied to analyze differential gene expressions ( $\left|\log _{2} \mathrm{FC}\right|>2 / 3$, adjusted $p$ Value 80.05$)$ in order to screen the target immune gene (imm-DEGs) among four subgroups of MB. The difference analysis results were visualized by using the ggplot2 and pheatmap package in the R software. Gene Ontology (GO) and Kyoto Encyclopedia of Genes and Genomes (KEGG) enrichment analysis were carried out using the clusterProfiler package in the $R$ software in order to annotate the biological function of imm-DEGs (adjusted $p$ Value $<0.05$ as statistically significant).

\subsection{Co-expression network construction.}

Weighted correlation network analysis (WGCNA) is a powerful algorithm that can be used for uncovering highly correlated genes with similar expression patterns, aiming to identify modules and genes related to disease phenotypes and therapeutic targets. The co-expression network was constructed using the WGCNA package in the $\mathrm{R}$ software and identified the genes with similar expression patterns. The key modules were gathered using the genes with similar expression patterns under the setting of $3 \mathrm{for}$ soft threshold power, 0.25 for the cut height, and 30 for the minimum module size.

2.5 Identification and correlation analysis of hub gene.

The threshold for hub genes was a significance (GS) $>0.4$ and Module Membership (MM) $>0.6$, based on the module constructed by co-expression network with WGCNA. Meanwhile, protein-protein interaction (PPI) network was constructed by applying a Search Tool for the Retrieval of Interacting Genes (STRING, https:// string-db.org/) database. Genes which have a degree of nodes in the PPI network $\geq 5$ were defined as hub genes of PPI network. Cytoscape software were applied to visualize PPI network. VennDiagram package in the $\mathrm{R}$ software was employed to compare the hub genes of modules with that of the PPI network. Then, corrplot and ggplot2 packages in the R software were also utilized to conduct a relevance analysis the immune hub gene and the differential immune infiltrating cells. 
2.6 The subgroup marker identification based on hub genes.

The subgroup marker was identified based on the immune hub gene expression pattern of four MB subgroups and then combined with the DNA methylation profiles to investigate the difference of the methylation level of the immune hub genes among four subgroups.

2.7 Validation of subgroups marker.

Dataset GSE37418 contained 73 MB samples which conclude 10 samples of SHH, 8 samples of WNT, 16 samples of Group 3, and 39 samples of Group 4, downloaded from GEO database. The expression pattern of the subgroup marker among the 4 subgroups were then compared and analyzed.

2.8 Construction of the multifactorial regulatory network based on the hub genes and the database.

The multifactorial regulatory network which consists of miRNA, IncRNA, and transcriptional factor (TF) was established through combining the hub genes with public databases such as the starBase, Harmonizome, etc. miRNA-mRNA and IncRNA-mRNA interaction network was applied by the starBase (http://starbase.sysu.edu.cn/, version 3.0), while mRNA-TF interaction network was applied by Harmonizome (http://maayanlab.cloud/Harmonizome/) database.

2.9 Immunochemistry staining:

CD4, CD31, S100A4, PRG2, GAB1, ABL1 and CXCR4 staining were performed with formalin-fixed, paraffinembedded medulloblastoma tissue microarrays. There are 5 samples in each group (SHH, WNT, Group 3, Group 4). The details of the subgroups and the patients for the tissue microarray were shown in Supplementary Data. Tissue microarrays were deparaffinized, rehydrated and washed three times with PBS. After performed antigen retrieval by soaked in sodium citrate $(\mathrm{pH6} .0)$ and heated to $100{ }^{\circ} \mathrm{C}$ for 15 min in the microwave oven, the tissue microarrays cooled to ambient temperature. Tissue microarrays were incubated with $3 \%$ hydrogen peroxide-methanol for 15 min and $10 \%$ goat serum with appropriate TritonX-100 in PBS for 30min for the sake of blocking the endogenous peroxidase and nonspecific binding respectively. They were incubated with CD4(1:150), CD31(1:1500), S100A4(1:250), PRG2(1:100), GAB1 (1:500), ABL1 (1:100) and CXCR4(1:400) antibodies overnight at $4^{\circ} \mathrm{C}$ and then washed with PBS, incubated with the appropriated secondary antibodies for 1 hour at ambient temperature. After stained with $D A B$ and counterstained with hematoxylin, the tissue microarrays were mounted and analyzed by the inverted microscopy (Zeiss Vert.A1, German).

\subsection{Statistical analysis}

All statistical analyses and graph generation were conducted with SPSS 23.0 (IBM, Armonk, NY, USA) and $\mathrm{R}$ software (R version 3.5.3; https://www.r-project.org/). Differences among groups were compared with one-way ANOVA. A p value of less than 0.05 was considered to be statistically significant.

\section{Results}


3.1 Difference of immune cell infiltration among medulloblastoma subgroups.

Tumor-infiltrating immune cells in medulloblastoma samples were examined with MethylCIBERSORT package in the $\mathrm{R}$ software based on the methylation data [12]. Based on the transcriptome expression data of tumor samples, estimate score, immune score and stromal score are estimated by using estimate according to the proportion of stromal and immune cells, which are used to predict tumor purity. The higher the estimated score, the lower the purity of tumor. The degree of immune cell infiltration, the immune scores, and purity of tumors of 763 samples in GSE85212 are displayed in Fig. 1A. It was evaluated by transcriptomic data within the same group of the samples from GSE85212. Variance analysis was employed to compare the degree of immune cell infiltration among different subtypes of $\mathrm{MB}$ and to establish the differential infiltrating immune cells. Results indicated that among the four subgroups of MB, 9 kinds of infiltrating immune cell (such as CD14, Treg, CD58, CD8, CD19, etc) were statistically significant in the degree of tumor infiltration (Figure. 1B) $(p<0.001)$.

3.2 Differential immune expressed gene and functional analysis among medulloblastoma subgroups.

Merging the immune gene sets from ImmPort and InnateDB databases, in total 2533 immune-related genes, consisting of 1794 immune gene from ImmPort immune gene set and 1052 immune gene from InnateDB immune gene set were included. Limma, a package for analyzing the differential expression, was utilized to screen the differentially expressed immune genes (imm-DEGs) among four subgroups of medulloblastoma. 293 immune genes that expressed differentially among four subgroups of medulloblastoma was established (adjusted $p$ Value $<0.05$ ). The differential gene distribution and heatmap of differential expression genes are illustrated in Fig. 2A-B.

The biological function enrichment analysis, including GO analysis and KEGG pathway enrichment analysis, was utilized. The $\mathrm{GO}$ analysis results emphasized that $293 \mathrm{imm}-\mathrm{DEGs}$ were associated with regulation of chemotaxis, regulation protein serine/threonine kinase activity, MAP kinase activity in the BP category(Figure.2C); receptor ligand activity, growth factor binding and cytokine receptor binding in the MF category(Figure.2D); and transcription factor complex, nuclear transcription factor complex, membrane microdomain in the CC category(Figure.2E); in the KEGG pathways, including RAS signaling pathway, Human cytomegalovirus infection, Chemokine signaling pathway, $T$ cell receptor signaling pathway, etc. were all implicated (Figure.2F).

3.3 Construction of the co-expression network based on imm-DEGs and identify key modules.

WGCNA, based on the imm-DEGs expression profiles, was employed to construct the co-expression network and distinguish the gene with similar expression pattern. A hierarchical clustering (Figure.3A) was used by setting the soft threshold power to 3 , cut height to 0.25 , and the minimum module size to 30 , to cluster the gene into different modules with different reprehensive color. In the end, four gene modules were established (Figure.3B). 
The degree of immune cell infiltration of samples was collected as a trait, and module-trait relationships were calculated according to the correlation in order to match the modules to their strongly related traits. As shown in Fig. 3C, the blue module was significantly related to Fibroblast cells, Eos cells and Endothelial cells, which are important components of immune microenvironment, and their interaction with tumor cells plays an important role in the growth of cancer (corr $=0.65,0.55,-0.6, p$ value $=5 e-92,1 e-$ $60,3 e-76$; respectively) and the correlation was stronger than other modules. Thus, blue was chosen to be the key module.

\subsection{Hub genes recognition.}

Genes from the selected blue key module were used to construct the PPI network, in which 18 hub genes were established by setting the node degree value $\geq 5$ (Figure.4A). 25 candidate hub genes from the blue module were identified by setting the gene significance $(G S)>0.4$ and module membership $(M M)>0.6$ (Figure.4B). Three immune hub genes (GAB1, ABL1, CXCR4) were obtained by synthesizing the hub genes in PPI network and the candidate hub genes in blue module. (Figure. $4 \mathrm{C}$ ).

The corrplot package in the R software was employed to investigate the correlation within the 3 hub genes. Results indicated that hub gene GAB1 was positively correlated with CXCR4 (Pearson corr $=0.77$ ); $A B L 1$ was negatively correlated with both CXCR4 and GAB1 (Pearson corr $=-0.55,-0.47$, respectively); these three hub genes were related with each other (Figure.4D).

The correlation between hub genes and the differential immune infiltrating cells were also scrutinized. As the result shows, both hub genes GAB1 and CXCR4 have a significant positive correlation with CD4_Eff and Fibroblast cells $(p<0.01)$, and a negative correlation with Endothelial cells and the Eos cells $(p<$ 0.001); while hub gene ABL1 has an opposite trend (Figure.4E). In addition, we selected the characteristic markers of these correlation immune cells, the immune hub genes and immune cells were confirmed by immunohistochemistry (Fig. 6B-C and Supplementary Figure.1). Due to the limited number of tissue samples, we will accumulate more samples for verification in the future.

3.5 Medulloblastoma subgroups markers recognition based on hub genes.

Three hub genes expression patterns in the subgroups of medulloblastoma were also studied and markers of the subgroups were recognized. It was indicated that immune hub gene GAB1 and CXCR4 express higher in the SHH subgroup than other three subgroups; immune hub gene ABL1 was expressed weakly in the SHH subgroup, while expressed strongly in other three subgroups (Figure.5A). The methylation level of immune hub genes among four subgroups was established by combining the immune hub gene with the DNA methylated data. The results of one-way ANOVA showed that the methylation levels of GAB1 and CXCR4 were significantly different among the four subtypes $(p<0.001)$ (Figure.5B).

3.6 Further verification of hub genes in independent data set and at histological level. 
GSE37418 was downloaded from GEO databases, which contained 73 medulloblastoma samples. GSE37418 consisted of 10 samples of SHH subgroup, 8 samples of WNT subgroup, 16 samples of Group 3 subgroup, and 39 samples of Group 4. Comparison and analysis of the expression patterns of subgroup markers were achieved. We found that the expression of GAB1 and CXCR4 was higher in SHH subtype than the other three subtypes, while ABL1 expressed lower in SHH subtype and higher in the other three subtypes (Figure.6A). Next, the immune hub genes were confirmed by immunohistochemistry (Fig. 6B-C). The expression of CXCR4 and GAB1 was apparently high in SHH subgroups compared with other subgroups. As Fig. 6B illustrates, the expression of ABL1 was the lower compared with CXCR4 and GAB1. Results of immunochemistry staining were consistent with the result of our bioinformatic analysis.

3.7 A multi-factor regulatory network of medulloblastoma based on immune hub genes.

A multi-factor regulatory network consisted of miRNA, IncRNA, and transcriptional factor (TF) was designed, combined with public databases (such as starBase, Harmonizome) and immune hub genes. Interactions among genes and their following products play a crucial role in many biological processes [13]. In the present study, a multi-factor network regarding hub genes combined with public databases (such as starBase, Haemonizome) was designed and constructed, which included miRNA, IncRNA, mRNA, and TF. Figure 7A shows the multifactor regulatory network of immune hub gene ABL1, GAB1 and CXCR4 in medulloblastoma. We found that CXCR4 is mainly involved in the regulation of IncRNA. GAB1 is related to the regulation of miRNA. ABL1 is not only involved in the regulation of IncRNA and miRNA, but also in the regulation of transcription factors. MiRNA, IncRNA and TF participate in the regulation of gene expression at the transcriptional and post transcriptional levels, and play a crucial role in gene expression. Therefore, they can be used as potential drug targets to indirectly regulate the expression of hub gene by acting on miRNA, IncRNA or TF.

\section{Discussion}

Medulloblastoma is the most frequent malignant pediatric brain tumor which originating from the posterior fossa. In the past 20 years, the standard treatment for medulloblastoma patients is surgery, radiotherapy and chemotherapy. The 5-year survival rate was $75 \%-85 \%$. One of the main complications of surgery is cerebellar reticence. Radiotherapy may cause severe adverse reactions such as cognitive function changes, vascular lesions, hearing loss and so on. Chemotherapy may have the risk of bone marrow suppression and organ dysfunction. There is an urgent need for more safety and effective adjuvant therapy for medulloblastoma. Tumor infiltrating immune cells have been proved to be the criteria for predicting the prognosis of solid tumors and predicting chemotherapy response [14]. The potential of immunotherapy for medulloblastoma has been discussed for many years [15]. However, despite the profound molecular characteristics of medulloblastoma, the progress of immunotherapy is slow, which may be due to the limited understanding of tumor microenvironment. Hence, we used bioinformatics method to quantify eight immune cell populations and two stromal cell populations as well as hub genes in a medulloblastoma dataset including 763 samples. 
Both transcriptional regulations and post-transcriptional regulations are essential regulatory mechanisms for cell cycle, including DNA replication, mitosis, and mitotic exit [16]. Gene regulatory network (GRN) refers to the network formed by the intercellular gene to gene interaction and it also refers specifically to the gene to gene interaction based on the regulation of gene expression. In addition, more and more researches focus on tumor infiltrating immune cells and related immunotherapy [15]. Previous study showed that a high number of activated cytotoxic T lymphocytes (CTLs) had a worse survival in MB patients [17]. Murata suggested that CD8 + tumor infiltrating lymphocytes are protective factors of $M B$ [18]. Using a deconvolution analysis based on methylation expression profiles, we firstly noticed immune infiltrating cells and two stromal cell populations that were statistically different among subgroups. According to the characteristics of microenvironment, Michael et al [19]also found significant differences in the aggregation of medulloblastoma subtypes. Former studies used method based on expression data such as CIBERSORT, TIMER, and ESTIMATE to approximate the abundance within the immune fraction and scores of enrichments, and they also performed low-resolution deconvolution [20,21]. However, distinguishing cellular subsets and their correlation would be more detailed with more intricate deconvolution using DNA methylation data [12]. Thus, MethyICIBERSORT was used first to derive estimates for different immune infiltrating cell populations among subgroups of medulloblastoma when both DNA methylation and gene expression data can be all obtained.

To investigate the immune infiltration of medulloblastoma, the GO and KEGG enrichment analyses were carried out to explore the biological function of imm-DEGs among subgroups of medulloblastoma and revealed that the overlapped DEGs were associated with chemokine signaling pathway. Chemokines and their receptors play an important role in physiological and pathological processes [22]. The relationship between chemokines and tumor biology is a subject of great concern in the scientific community. Significant results have been achieved in the treatment of MB by using chemokines and their receptors [23], combined with our analysis, it indicates the important role of chemokines in MB and the possible therapeutic ability.

The module identified by WGCNA is highly related to a certain personality, and the genes that make up this module are likely to participate in a certain pathway or biological process; through the construction of PPI network and the identification of hub gene, we can further screen out some genes that may play a key role in this process, so as to provide a more accurate identification of subtype marker [24], therefore WGCNA were used to identify the gene modules that are specifically associated with similar expression patterns in medulloblastomas. A total of 294 immune differential expressed genes were investigated and categorized into 5 modules. The blue module was chosen as the key module and included in the construction of PPI network based on its significant correlation with the trait of immune infiltration. According to the results of the blue module and the PPI network, we have identified three hub genes: CXCR4, GAB1, and ABL1 as immune infiltration markers among subgroups of medulloblastoma; a multifactor regulatory network was also set up based on the three hub genes.

Sengupta et al found that CXCR4 only exhibited overexpression in the SHH-MBs when compared with normal high levels found in the fetal cerebellum, and it was essential for SHH pathway activation in 
medulloblastoma [25]. It has been demonstrated by Yang et al that the inhibition of CXCR4 blocked the intracranial growth of medulloblastoma cell lines [26]. Similarly, Ward et al. combined CXCR4 and SHH pathway antagonism, which produced an enhanced therapeutic effect[27]. In the present study, CXCR4 was highly expressed significantly in SHH-MBs compared with the other three subgroups, which is encouraging as this confirmed the close relationship between CXCR4 and SHH at the transcriptional level. Also, CXCR4 was associated with immune response of other tumors, and anti-CXCR4 union and anti-PD-1 immunotherapies are used in treating other CNS tumors, which further confirmed that CXCR4 is related to immune infiltration [28]. GAB1 (GRB2- associated binding protein 1), a member of GAB/DOS family of adapter proteins, is tyrosine-phosphorylated when stimulated by various growth factors and cytokines [29]. Immunoreactivity for GAB1 was found specific in SHH-MBs in a cohort $(n=235)$ of medulloblastoma patients aged between 0.4 and 52 years [30]. Although the role of GAB1 in the SHH pathway in medulloblastomas and other central system tumors is still not elucidated, the current study discovered a higher expression of GAB1 in SHH-MB compared with other subgroups, which is consistent with the above literature. $A B L 1$, a non-receptor tyrosine kinase, is involved in various biological functions. Zhu et al identified that $A B L 1$ with other 11 genes can construct a prognostic nomogram predicting the overall survival for medulloblastoma patients [31]. In our study, ABL1 was initially thought to be expressed lowly in SHH-MB, while high in the other three subgroups.

It is unfortunate that the present study has not explored the multi-factor regulatory network constructed by hub gene in-depth. This is due to that the lack of clarification on the mechanism of the three hub genes, leaving us room for further investigation.

In conclusion, the present study has proposed bioinformatic results of the inn-DEGs, three immune hub genes (CXCR4, GAB1, ABL1), and the multi-factor regulatory network from datasets of GEO and immune gene profiles by algorithms (melthyCIBERSORT, WGCNA), PPI network, and public databases (starBase, Harmonizome). The three immune hub genes with differential expressions among subgroups of medulloblastoma emphasize the heterogenicity of the tumor immune infiltration microenvironment in medulloblastoma subgroups. With the rapid development of high-throughput analysis, molecular stratifications are gradually becoming the main trend in molecular targeted therapy and prognostic risk assessment model. Our research is just a step towards exploring tumor immune infiltration and provides the framework for identification of immune infiltration marker and multi-factor regulatory network in medulloblastomas. The analysis based on immune difference has important clinical significance for molecular typing and immunotherapy. However, there are still some limitations in this study, transcriptome analysis only reflects some aspects of the immune state, rather than the overall changes. We will further explore in the follow-up study.

\section{Declarations}

\section{Funding}


This research was funded by The National Natural Science Foundation of China, grant number 81972338. This study was also funded by Advanced Research and Training Program of Beijing Double Leading Scholars from China academy of Chinese Medical Science. This research was also supported by Clinical Major Specialty Projects of Beijing.

\section{Acknowledgments}

We would like to thank China National Clinical Research Center for Neurological Diseases and Chinese Medical Science for administrative support.

\section{Author contribution}

W. Li and F. Chen were involved in conceptualization. S. Li designed the research and guided the experiments. C. Wang, L.Pan and Z. Cai was involved in analyzed and interpreted the data. S. Li and J. Chen were involved in the experimental validation. J. Chen. was involved in formal writing. S. Li and F. Chen were involved in review and editing. F. Chen was involved in supervision. W. Li was involved in funding acquisition. All authors contributed to the article and agreed to the published version of the manuscript.

\section{Conflict of Interest}

The authors declare that the research was conducted in the absence of any commercial or financial relationships that could be construed as a potential conflict of interest.

\section{Ethics approval and consent to participate}

The study was approved by the Institutional Review Boards of Beijing Tiantan Hospital (Beijing, China). The procedures of the human tissues collection were reviewed and approved by the Ethics Committee of Beijing Tiantan Hospital, Capital Medical University [approval no. 2021-020-01].

\section{Data Availability Statement}

The datasets presented in this study can be found in online repository. The names of the repository and accession number is GSE85218.

\section{References}


1. Louis DN, Perry A, Reifenberger G, von Deimling A, Figarella-Branger D, Cavenee WK, Ohgaki H, Wiestler OD, Kleihues P, Ellison DW: The 2016 World Health Organization Classification of Tumors of the Central Nervous System: a summary. Acta Neuropathologica 2016, 131(6):803-820.

2. Pomeroy SL, Tamayo P, Gaasenbeek M, Sturla LM, Angelo M, McLaughlin ME, Kim JY, Goumnerova LC, Black PM, Lau C et al: Prediction of central nervous system embryonal tumour outcome based on gene expression. Nature 2002, 415(6870):436-442.

3. Taylor MD, Northcott PA, Korshunov A, Remke M, Cho YJ, Clifford SC, Eberhart CG, Parsons DW, Rutkowski S, Gajjar A et al: Molecular subgroups of medulloblastoma: the current consensus. Acta Neuropathol 2012, 123(4):465-472.

4. Ellison DW, Kocak M, Dalton J, Megahed H, Lusher ME, Ryan SL, Zhao W, Nicholson SL, Taylor RE, Bailey $S$ et al: Definition of disease-risk stratification groups in childhood medulloblastoma using combined clinical, pathologic, and molecular variables. J Clin Oncol 2011, 29(11):1400-1407.

5. Northcott PA, Korshunov A, Witt H, Hielscher T, Eberhart CG, Mack S, Bouffet E, Clifford SC, Hawkins CE, French $P$ et al: Medulloblastoma comprises four distinct molecular variants. J Clin Oncol 2011, 29(11):1408-1414.

6. Ecker J, Oehme I, Mazitschek R, Korshunov A, Kool M, Hielscher T, Kiss J, Selt F, Konrad C, Lodrini M et al: Targeting class I histone deacetylase 2 in MYC amplified group 3 medulloblastoma. Acta Neuropathol Commun 2015, 3:22.

7. Kool M, Korshunov A, Remke M, Jones DT, Schlanstein M, Northcott PA, Cho YJ, Koster J, Schoutenvan Meeteren A, van Vuurden D et al: Molecular subgroups of medulloblastoma: an international meta-analysis of transcriptome, genetic aberrations, and clinical data of WNT, SHH, Group 3, and Group 4 medulloblastomas. Acta Neuropathol 2012, 123(4):473-484.

8. Zhao F, Ohgaki H, Xu L, Giangaspero F, Li C, Li P, Yang Z, Wang B, Wang X, Wang Z et al: Molecular subgroups of adult medulloblastoma: a long-term single-institution study. Neuro Oncol 2016, 18(7):982-990.

9. Hirata E, Sahai E: Tumor Microenvironment and Differential Responses to Therapy. Cold Spring Harb Perspect Med 2017, 7(7).

10. Binnewies M, Roberts EW, Kersten K, Chan V, Fearon DF, Merad M, Coussens LM, Gabrilovich DI, Ostrand-Rosenberg S, Hedrick CC et al: Understanding the tumor immune microenvironment (TIME) for effective therapy. Nat Med 2018, 24(5):541-550.

11. Northcott PA, Buchhalter I, Morrissy AS, Hovestadt V, Weischenfeldt J, Ehrenberger T, Grobner S, Segura-Wang M, Zichner T, Rudneva VA et al: The whole-genome landscape of medulloblastoma subtypes. Nature 2017, 547(7663):311-317.

12. Chakravarthy A, Furness A, Joshi K, Ghorani E, Ford K, Ward MJ, King EV, Lechner M, Marafioti T, Quezada SA et al: Pan-cancer deconvolution of tumour composition using DNA methylation. Nat Commun 2018, 9(1):3220.

13. Swank Z, Laohakunakorn N, Maerkl SJ: Cell-free gene-regulatory network engineering with synthetic transcription factors. Proc Natl Acad Sci U S A 2019, 116(13):5892-5901. 
14. Fridman WH, Pagès F, Sautès-Fridman $C$, Galon J: The immune contexture in human tumours: impact on clinical outcome. Nat Rev Cancer 2012, 12(4):298-306.

15. Sonabend AM, Ogden AT, Maier LM, Anderson DE, Canoll P, Bruce JN, Anderson RC: Medulloblasoma: challenges for effective immunotherapy. J Neurooncol 2012, 108(1):1-10.

16. Romanel A, Jensen LJ, Cardelli L, Csikasz-Nagy A: Transcriptional regulation is a major controller of cell cycle transition dynamics. PLoS One 2012, 7(1):e29716.

17. Vermeulen JF, Van Hecke W, Adriaansen EJM, Jansen MK, Bouma RG, Villacorta Hidalgo J, Fisch P, Broekhuizen R, Spliet WGM, Kool M et al: Prognostic relevance of tumor-infiltrating lymphocytes and immune checkpoints in pediatric medulloblastoma. Oncoimmunology 2018, 7(3):e1398877.

18. Murata D, Mineharu Y, Arakawa Y, Liu B, Tanji M, Yamaguchi M, Fujimoto KI, Fukui N, Terada Y, Yokogawa $\mathrm{R}$ et al: High programmed cell death 1 ligand-1 expression: association with CD8 + T-cell infiltration and poor prognosis in human medulloblastoma. J Neurosurg 2018, 128(3):710-716.

19. Bockmayr M, Mohme M, Klauschen F, Winkler B, Budczies J, Rutkowski S, Schüller U: Subgroupspecific immune and stromal microenvironment in medulloblastoma. Oncoimmunology 2018, 7(9):e1462430.

20. Aran D, Sirota M, Butte AJ: Systematic pan-cancer analysis of tumour purity. Nat Commun 2015, 6:8971.

21. Newman AM, Liu CL, Green MR, Gentles AJ, Feng W, Xu Y, Hoang CD, Diehn M, Alizadeh AA: Robust enumeration of cell subsets from tissue expression profiles. Nat Methods 2015, 12(5):453-457.

22. Hughes CE, Nibbs RJB: A guide to chemokines and their receptors. Febs j 2018, 285(16):2944-2971.

23. Ozawa PM, Ariza CB, Ishibashi CM, Fujita TC, Banin-Hirata BK, Oda JM, Watanabe MA: Role of CXCL12 and CXCR4 in normal cerebellar development and medulloblastoma. Int J Cancer 2016, 138(1):10-13.

24. Langfelder $\mathrm{P}$, Horvath S: WGCNA: an R package for weighted correlation network analysis. $B M C$ Bioinformatics 2008, 9:559.

25. Sengupta R, Dubuc A, Ward S, Yang L, Northcott P, Woerner BM, Kroll K, Luo J, Taylor MD, WechslerReya RJ et al: CXCR4 activation defines a new subgroup of Sonic hedgehog-driven medulloblastoma. Cancer Res 2012, 72(1):122-132.

26. Yang L, Jackson E, Woerner BM, Perry A, Piwnica-Worms D, Rubin JB: Blocking CXCR4-mediated cyclic AMP suppression inhibits brain tumor growth in vivo. Cancer Res 2007, 67(2):651-658.

27. Ward SA, Warrington NM, Taylor S, Kfoury N, Luo J, Rubin JB: Reprogramming MedulloblastomaPropagating Cells by a Combined Antagonism of Sonic Hedgehog and CXCR4. Cancer Res 2017, 77(6):1416-1426.

28. Wu A, Maxwell R, Xia Y, Cardarelli P, Oyasu M, Belcaid Z, Kim E, Hung A, Luksik AS, Garzon-Muvdi T et al: Combination anti-CXCR4 and anti-PD-1 immunotherapy provides survival benefit in glioblastoma through immune cell modulation of tumor microenvironment. J Neurooncol2019, 143(2):241-249. 
29. Anatoly Kiyatkin EA, Nick I. Markevich, Nikolay M. Borisov, Jan B. Hoek, and Boris N. Kholodenko: Scaffolding protein Grb2-associated binder 1 sustains epidermal growth factor-induced mitogenic and survival signaling by multiple positive feedback loops. The journal of biological chemistry 2006, 281:19925-19938.

30. Ellison DW, Dalton J, Kocak M, Nicholson SL, Fraga C, Neale G, Kenney AM, Brat DJ, Perry A, Yong WH et al: Medulloblastoma: clinicopathological correlates of SHH, WNT, and non-SHH/WNT molecular subgroups. Acta Neuropatho/ 2011, 121(3):381-396.

31. Zhu S, Lin F, Chen Z, Jiang X, Zhang J, Yang Q, Chen Y, Wang J: Identification of a Twelve-Gene Signature and Establishment of a Prognostic Nomogram Predicting Overall Survival for Medulloblastoma. Front Genet 2020, 11:563882.

\section{Figures}


A

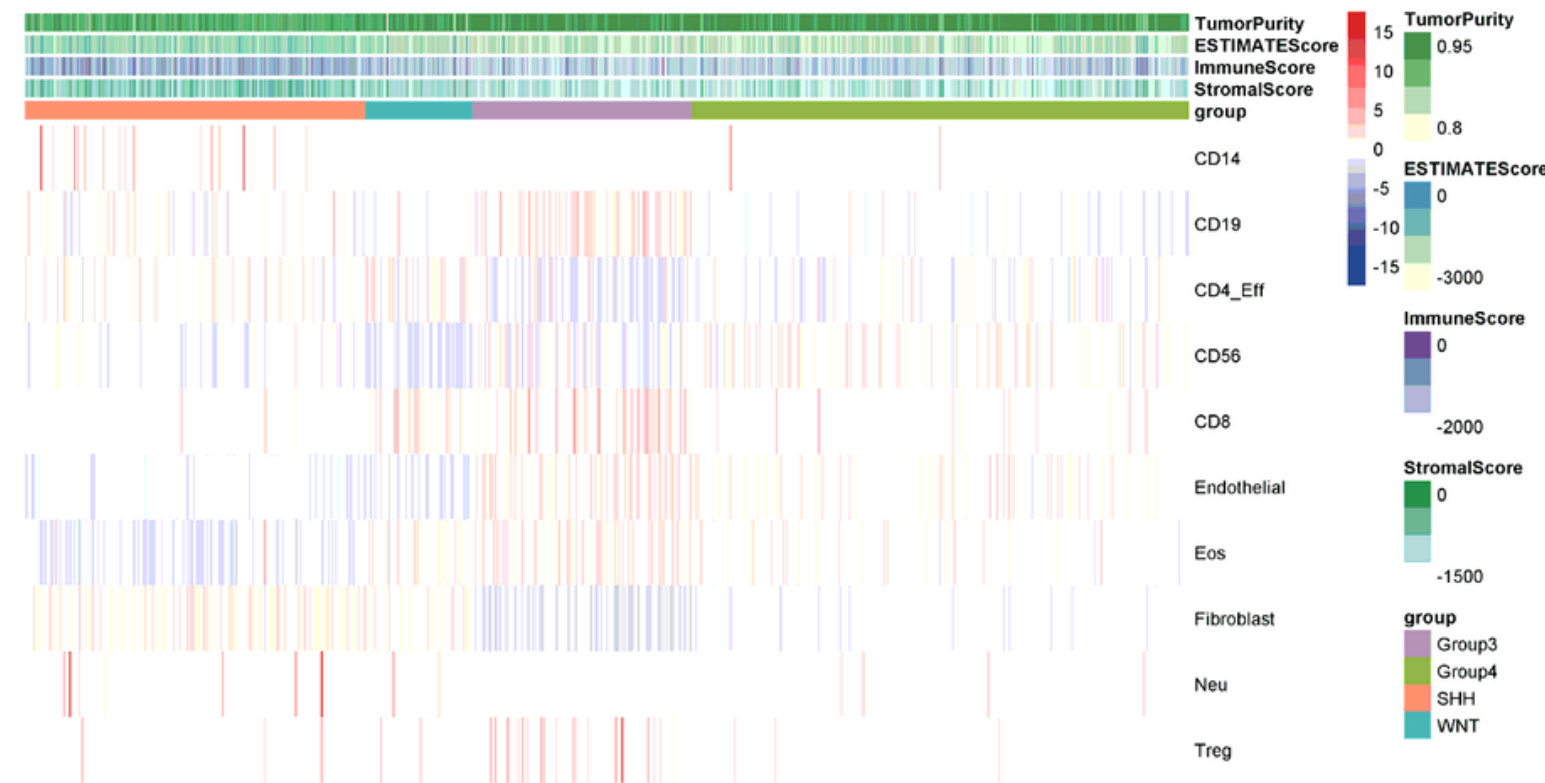

B

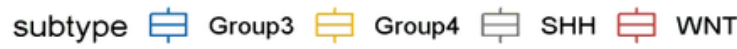



Figure 1

(A)The infiltration degree of infiltrating cells in medulloblastoma samples was evaluated by MethyICIBERSORT deconvolution. The abscissa represents medulloblastoma samples, and the ordinate represents the cells existing in tumor samples for identification. (B) The differences of immune cell infection along four subgroups of medulloblastoma were analyzed by one-way ANOVA. Abscissa: the 
infiltrating cells identified in tumor samples; ordinate: the proportion of each type of cells in tumor tissue. (ns: $p>0.05, *: p<=0.05, * *: p<=0.01, * \star *: p<=0.001, * \star \star *: p<=0.0001$ ).
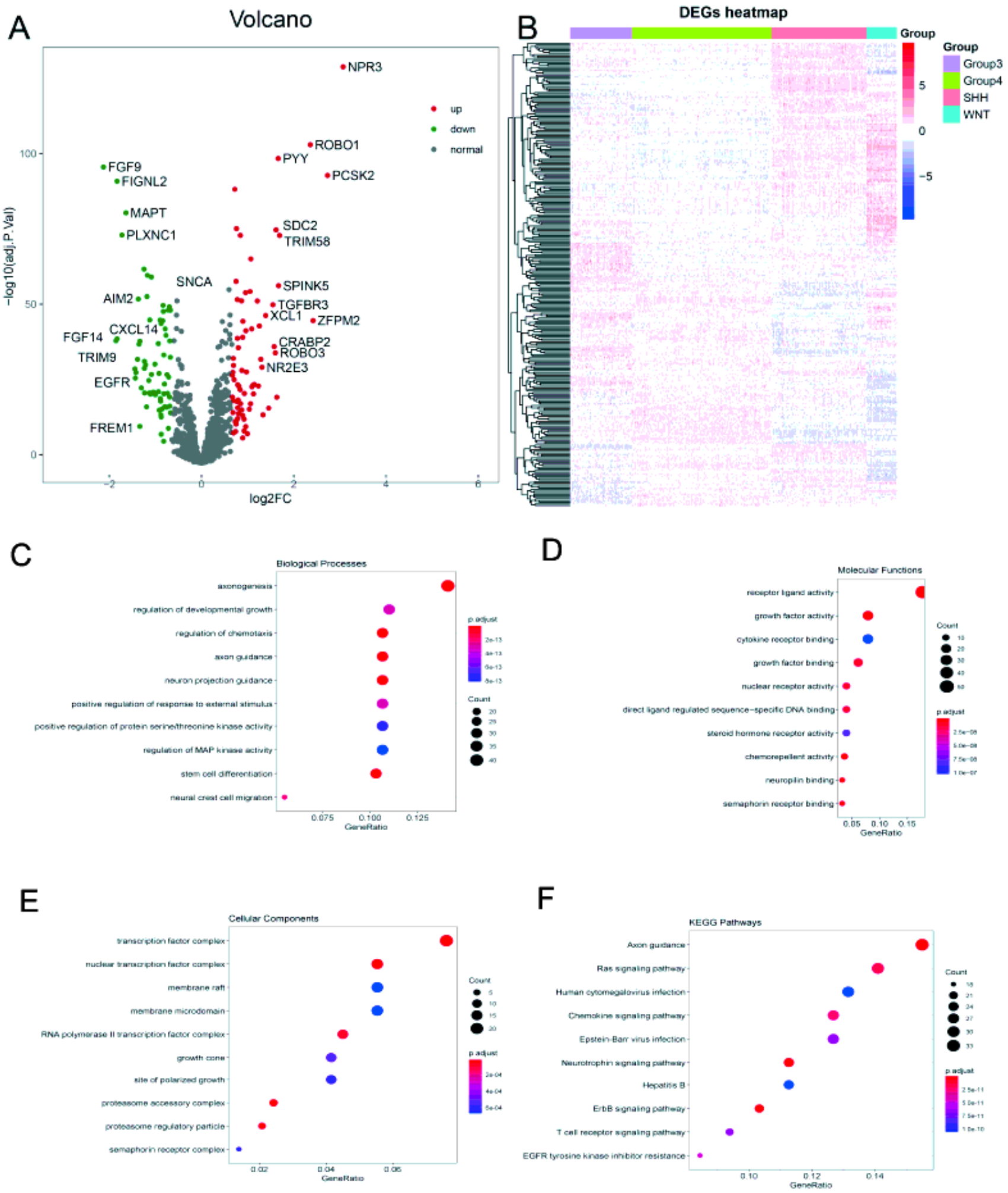

Figure 2

GO and KEGG enrichment analysis of imm-DEGs. (A) Distribution of differential expressed genes in group 3 vs. group 4 are depicted by the volcano plot. (B) The differentially expressed immune genes clustered in the four subgroups of medulloblastoma. (C) imm-DEGs were enriched in the biological processes. (D) 
Molecular functions of gene ontology. (E) Cellular components of gene ontology. (F) KEGG enrichment analysis of imm-DEGs.

A

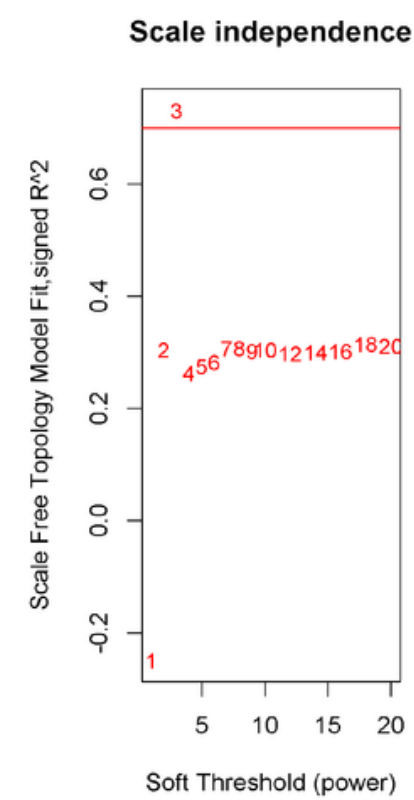

C
B



\section{Module-Trait Relationships}

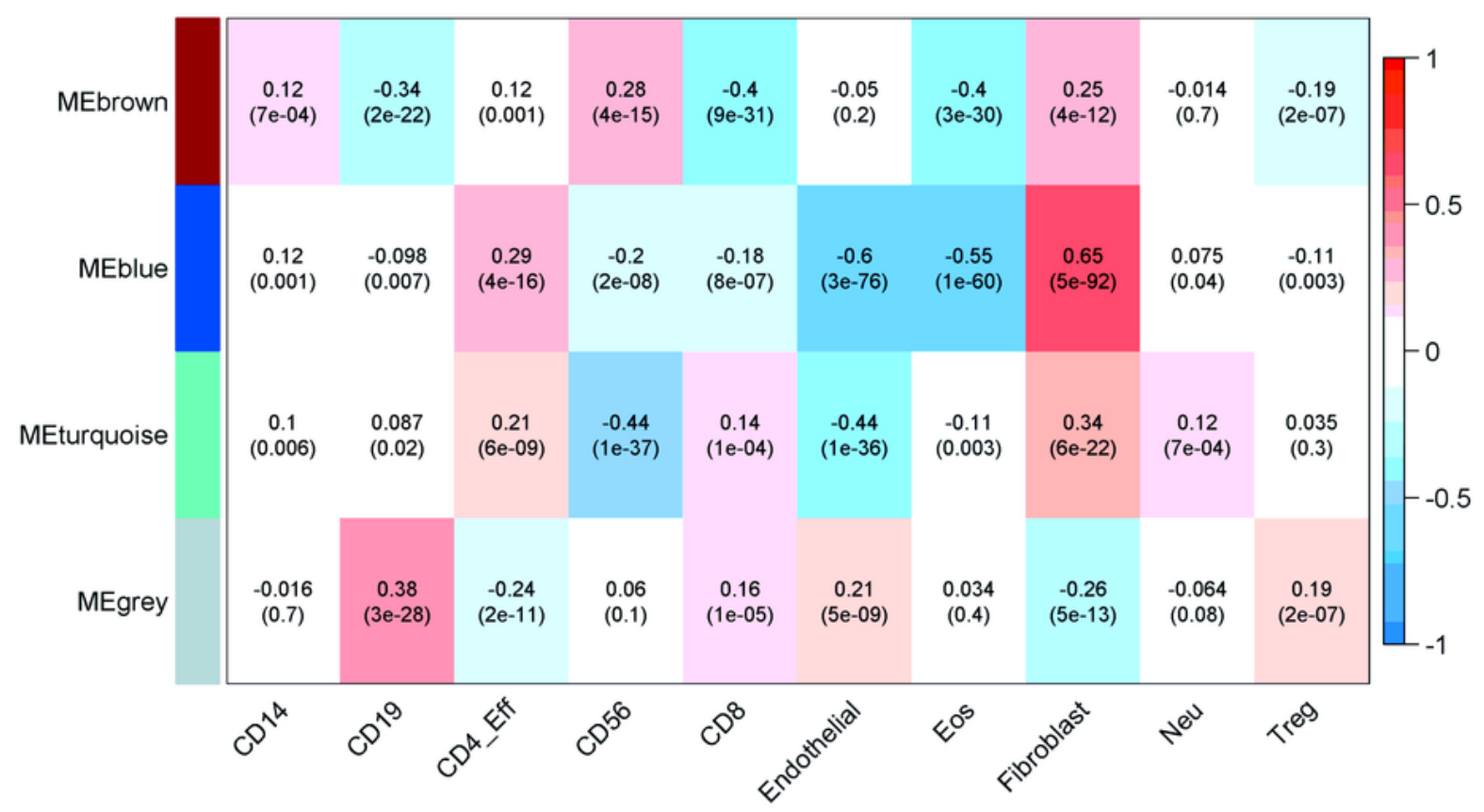

Figure 3

Construction of the co-expression network. (A) A hierarchical clustering was used by setting the soft threshold power to 3 , cut height to 0.25 , and the minimum module size to 30 , scale independence and mean connectivity analysis; (B) Cluster the gene into different modules with different reprehensive color 
among different modules. (C) The degree of immune cell infiltration of samples was collected and module-trait relationships were calculated according to the correlation.
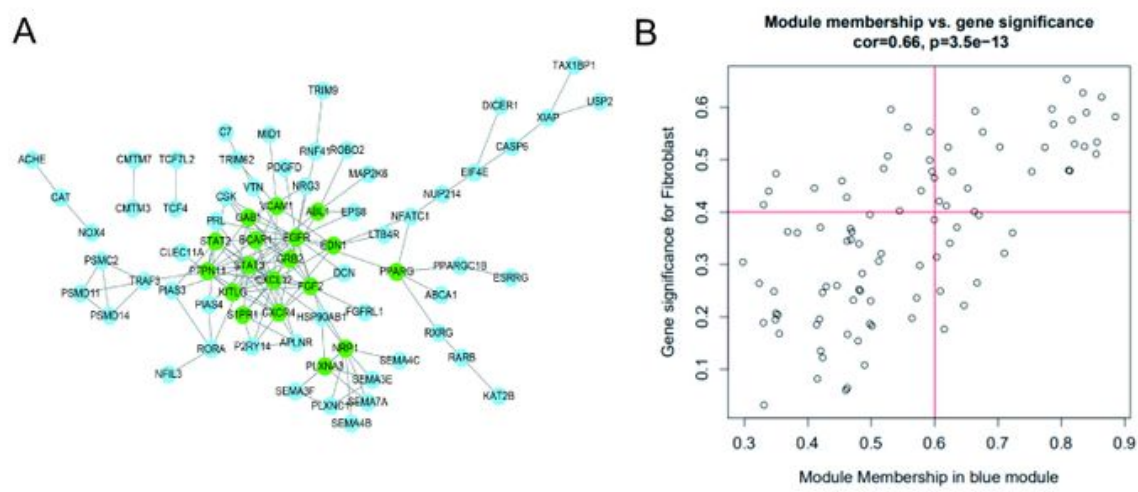

C
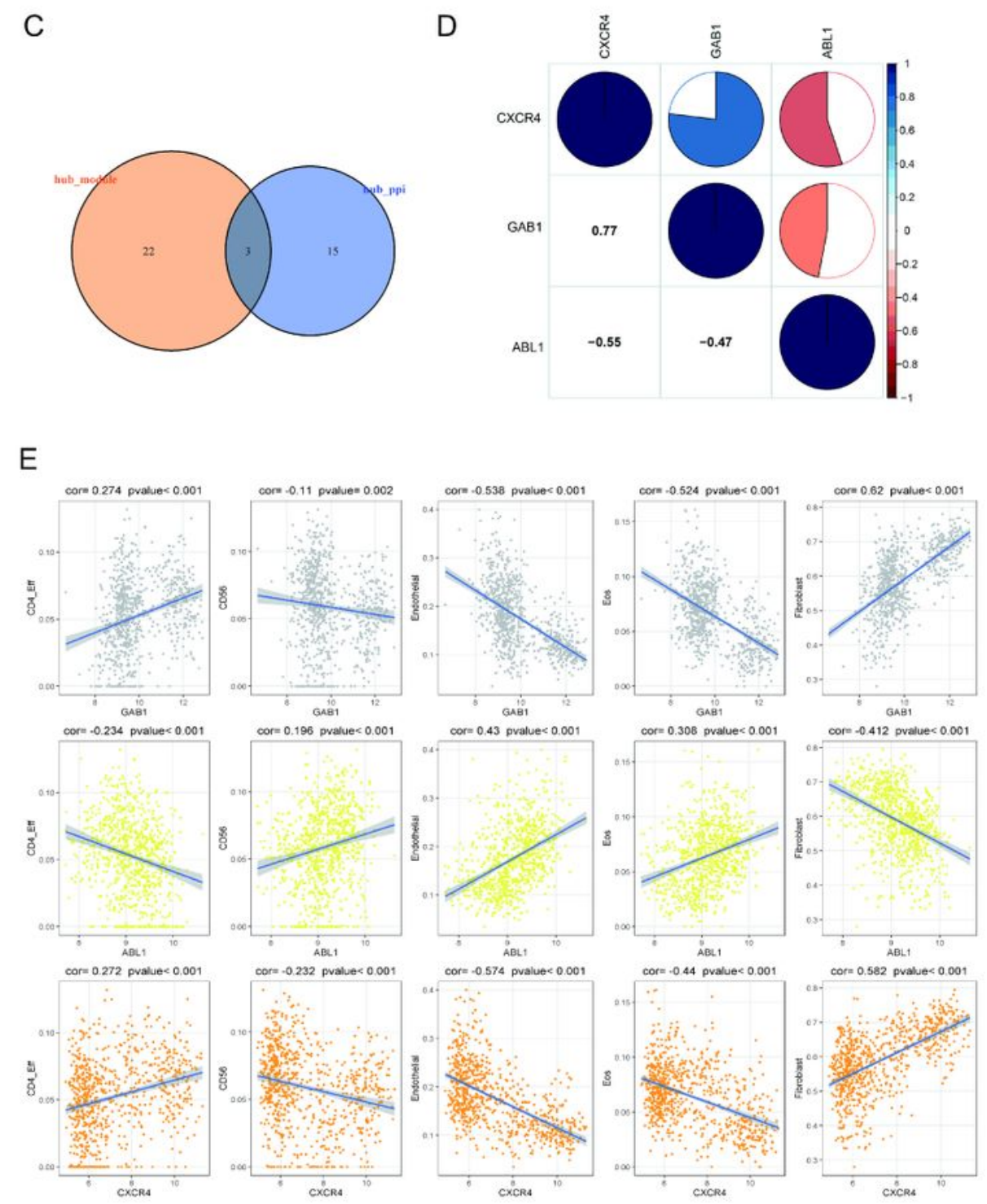

Figure 4

The correlation analysis of hub gene and its relationship with different immune infiltrating cells. (A) The PPI network was constructed by genes in the blue module. The nodes with a green color of PPI network represent the hub genes. (B) The distribution of gene significance (GS) and module membership (MM) in 
the blue module. (C) The intersection of immune hub gene set of PPI network (node degree value $\geq 5$ ) and hub gene set of blue modules ( $G S>0.4, M M>0.6$ ). (D) Correlation analysis of four hub genes. Blue represents positive correlation and red represents negative correlation. The larger the sectorial area of the pie graph, the higher the correlation between the two genes. $(E)$ The correlation between hub genes (GAB1, ABL1, CXCR4) and immune infiltrating cells (CD4_Eff, Fibroblast cells, Endothelial cells and the Eos cells).

A
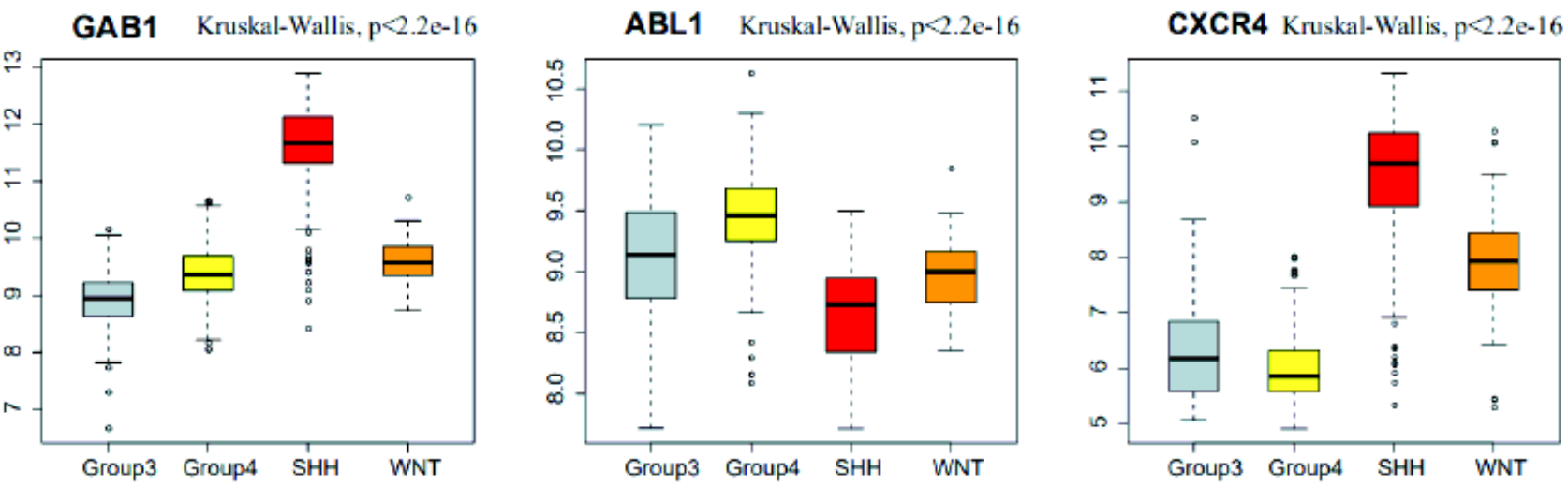

B

subtype 追 Group3 官 Group4 户 SHH 官 WNT



Figure 5 
Medulloblastoma subgroups markers recognition based on hub genes. (A) Three hub genes expression patterns in the subgroups of medulloblastoma. (B) The methylation level of immune hub genes among four subgroups was established by combining the immune hub gene with the DNA methylated data. (ns: $p>0.05, *: p<=0.05, * *: p<=0.01, * * *: p<=0.001, * * * *: p<=0.0001)$.

A
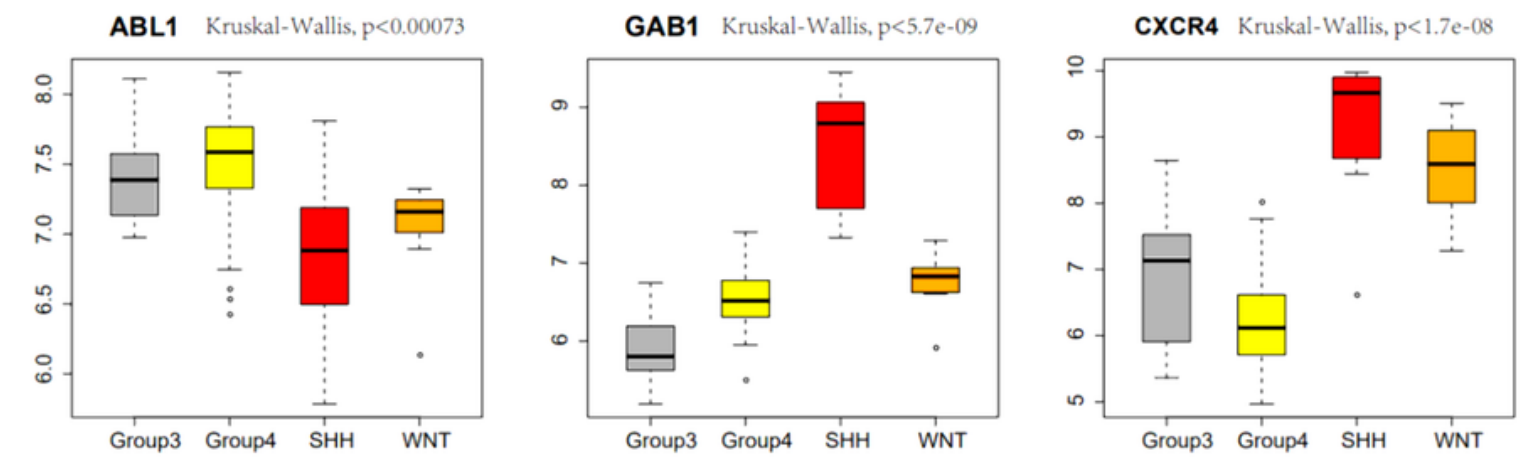

B Group3 Group4 $\mathrm{SHH}$ WNT

ABL1


GAB1

CXCR4


C
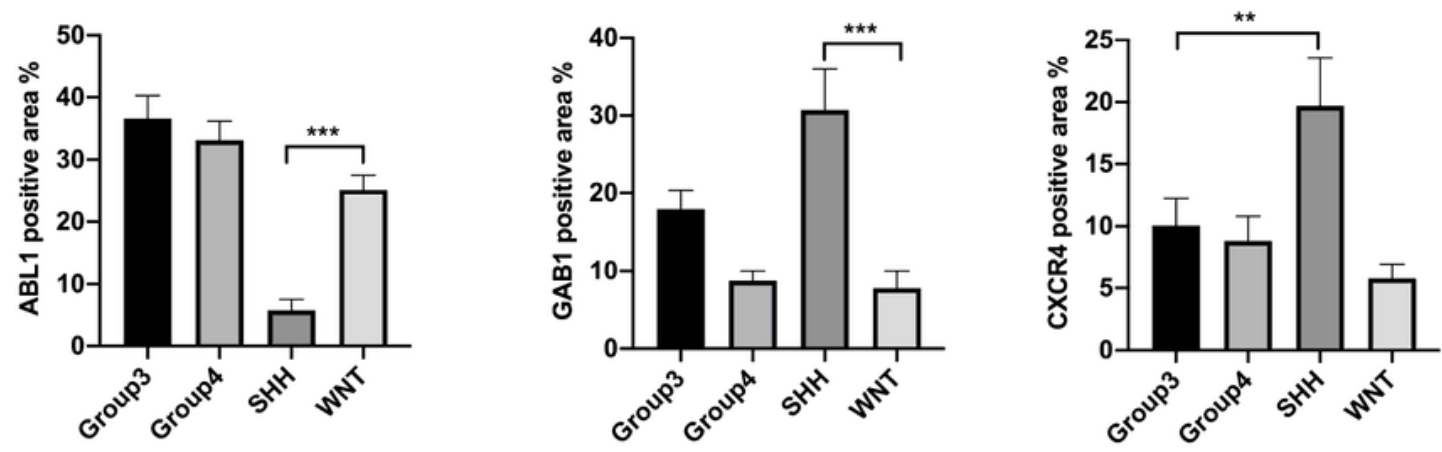

Figure 6 
Further verification of hub genes. (A) The expression of GAB1, ABL1and CXCR4 in different subtypes from independent data set. (B) Further verification by immunohistochemistry $(n=5)(* *: p<=0.01, \star \star \star$ : $p$ $<=0.001)$. (C) Average positive stained area percentage of ABL1, GAB1 and CXCR4.



\section{Figure 7}

The medulloblastoma multi-factor regulatory network based on immune hub genes. A. The multifactor regulatory network of immune hub gene ABL1, GAB1 and CXCR4 in medulloblastoma, each node represents a gene; red circles represent mRNA, yellow diamonds represent TF, blue triangles represent IncRNA, and orange arrows represent miRNA.

\section{Supplementary Files}

This is a list of supplementary files associated with this preprint. Click to download.

- supplementary20210713.docx 